Hooper and colleagues, therefore, investigated the efficacy and tolerability of once-weekly $280 \mathrm{mg}$ alendronate sodium taken as an oral buffered solution (OBS).

A total of 63 patients with PDB (mean age 69.3 years) were enrolled at clinical centers in Europe, North America, Australasia and Africa. Participants received alendronate sodium in a regimen of either a $40 \mathrm{mg} /$ day tablet $(n=21)$ or a $280 \mathrm{mg}$ OBS once weekly $(n=42)$ for 6 months; 52 patients completed the study (19 in the tablet group, 33 in the OBS group). At 6 months, total serum alkaline phosphatasea marker for PDB-decreased from baseline by $73 \%$ and $72 \%$ in the tablet and OBS treatment groups, respectively. The incidence of non-serious adverse events was similar in both treatment groups, although more patients in the $280 \mathrm{mg}$ once-weekly group discontinued treatment because of adverse events than patients in the $40 \mathrm{mg}$ once-daily group (19.0\% vs $9.5 \%)$.

The authors commented that, although the two dosing regimens have similar efficacies, the $40 \mathrm{mg} /$ day tablet was better tolerated. They did, however, add that "the potential for improved esophageal safety of alendronate sodium $280 \mathrm{mg}$ once-weekly OBS should not be ignored".

Original article Hooper M et al. (2008) Randomized, activecontrolled study of once-weekly alendronate $280 \mathrm{mg}$ high dose oral buffered solution for treatment of Paget's disease. Osteoporos Int [doi:10.1007/s00198-008-0639-6]

\section{Multitarget therapy for lupus nephritis with diffuse proliferative and membranous lesions}

Patients with severe lupus nephritis who have both diffuse proliferative (class IV) and membranous (class $V$ ) lesions on biopsy can be refractory to current monotherapy treatments. Bao et al. compared mycophenolate mofetil, tacrolimus and corticosteroid multitarget treatment with cyclophosphamide in patients with class V+IV lupus nephritis in order to determine an optimized immunosuppressive strategy for these individuals.

This Chinese, open-label study enrolled 40 patients with class $V+$ IV lupus nephritis who were randomly allocated to receive induction therapy with either intravenous cyclophosphamide pulse therapy or mycophenolate mofetil and tacrolimus for 6 months, or for 9 months if complete remission was not achieved. All patients also received intravenous methylprednisolone pulse therapy for the first 3 days of the study and oral prednisone throughout.

In intention-to-treat analyses, significantly more patients in the multitarget therapy group than in the cyclophosphamide group achieved complete or partial remission at 6 months (18 [90\%] vs 9 [45\%]; $P=0.002)$ and at 9 months (19 [95\%] vs 11 [55\%]; $P=0.003$ ). The overall incidence of adverse effects (e.g. gastrointestinal syndrome and leukopenia) was lower in the multitarget therapy group than in the cyclophosphamide group, although one patient in each group developed pneumonia.

The authors conclude that this multitarget therapy regimen is better at inducing remission than is intravenous cyclophosphamide in patients with both diffuse proliferative and membranous lupus nephritis. This efficacy could be due to the synchronous effects of the different components of the multitarget therapy on the different types of lesions in severe lupus nephritis.

Original article $\mathrm{Bao} \mathrm{H}$ et al. (2008) Successful treatment of class $\mathrm{V}+\mathrm{IV}$ lupus nephritis with multitarget therapy. J Am Soc Nephrol [doi:10.1681/ASN.2007121272]

\section{Etanercept is suitable for children with JIA under the age of 4 years}

Etanercept is the only biologic agent approved for treating children with juvenile idiopathic arthritis (JIA) who are over the age of 4 years with a disease course refractory to methotrexate treatment. Data is lacking, however, on the efficacy and safety of etanercept in patients younger than 4 years of age. Tzaribachev et al. have, therefore, performed an analysis of data on children in this subgroup from the German JIA Etanercept Registry.

The study identified 25 patients under the age of 4 years treated with etanercept (mean duration 19 months, range 1-54 months), of whom 15 had systemic-onset JIA (soJIA) and 10 had nonsystemic JIA (nsJIA). Seven patients with soJIA and nine with nsJIA met the American College of Rheumatology pediatric response criteria for $70 \%$ improvement from baseline, while five children with soJIA and two with nsJIA achieved a complete response. 